\title{
Klassenmanagement bei angehenden Lehrkräften aufbauen
}

\section{Eine Seminarevaluation unter Berücksichtigung von Überzeugungen und Selbstwirksamkeit bezüglich inklusiver Bildung}

\section{Marie-Luise Gehrmann \& Satyam Antonio Schramm}

Journal für Psychologie, 27(2), 95-116

https://doi.org/10.30820/0942-2285-2019-2-95

www.journal-fuer-psychologie.de

\section{Zusammenfassung}

Ein effizientes Klassenmanagement ist eine notwendige Bedingung hochwertigen, inklusiven Unterrichts. Der Aufbau der dazu notwendigen Kompetenzen ist ein Desiderat in der LehrerInnenbildung. Ein darauf zielendes Seminarkonzept mit videogestütztem Micro-teaching für Lehramtsstudierende im Praxissemester wird vorgestellt und evaluiert. Abgeleitet aus dem Modell professioneller Handlungskompetenz von Lehrkräften wird dabei untersucht, inwiefern berufsbezogene Überzeugungen und Selbstwirksamkeitsüberzeugungen bezüglich inklusiver Bildung den Aufbau und die Anwendung von Klassenmanagementstrategien begünstigen können. Im Ergebnis der Analyse zweier Interventionsdurchgänge mit insgesamt 35 Studierenden in der Interventions- und 34 Studierenden in der Vergleichsgruppe deuten die Daten in die erwartete Richtung, belegen jedoch nur für Performanzen im Verhaltensmanagement mittlere Effekte. Die Überzeugungen bezüglich inklusiver Pädagogik blieben bei allen Studierenden relativ stabil. Die Selbstwirksamkeitsüberzeugungen zur Umsetzung inklusiver Unterrichtspraxis nahmen zum Ende des Praxissemesters zu. Ihre Bedeutung für die Interventionseffekte erscheint nicht eindeutig.

Schlüsselwörter: Lehrerbildung, Klassenmanagement, Einstellungen, Überzeugungen, Selbstwirksamkeit, Evaluation, Micro-teaching

\section{Summary}

Establishing classroom management for prospective teachers. A seminar evaluation considering beliefs and self-efficacy in inclusive teaching

Efficient classroom management is a necessary condition of high-quality, inclusive teaching. Thus, the development of essential competences is a desideratum in teacher education. A seminar concept based on video-assisted micro-teaching for students in their practical se- 
mester is presented and evaluated. Derived from the model of professional competence of teachers, we examine how job-related and self-efficacy beliefs regarding inclusive education can foster the development and application of classroom management strategies. Two intervention-phases with a total of 35 students in the treatment and 34 students in the control group were carried out. Obtained data point in the expected direction but only show medium effects for performance in behavior management. Beliefs concerning inclusive education remained relatively stable among all students. Moreover, self-efficacy for inclusive teaching practices increased at the end of the practical semester. The influence on intervention effects does not seem clear.

Key words: teacher education, classroom management, attitudes, beliefs, self-efficacy, evaluation, micro-teaching

Seit der bildungspolitischen Verpflichtung zur Umsetzung eines inklusiven Bildungssystems eines Großteils der EU-Mitgliedsstaaten erscheint die Diskussion um die Dimensionen und die Genese professioneller Handlungskompetenz von Lehrkräften besonders lebhaft. Baumert und Kunter (2006) entwickelten ein heuristisches Modell professioneller Handlungskompetenz von Lehrkräften, welches einen metatheoretischen Rahmen zur Verortung empirischer Befunde sowie ihre theoriebezogene Erläuterung bereitstellt. Es umfasst Professionswissen (z. B. pädagogisches Wissen, Fachwissen, fachdidaktisches Wissen), Werthaltungen und Überzeugungen (z. B. subjektive Theorien über Lehren und Lernen) sowie in Bezug auf die psychologische Funktionsfähigkeit der handelnden Lehrkräfte motivationale Orientierungen und Selbstregulation (vgl. Abbildung 1). An jene Konzeptualisierung knüpft die hier vorliegende Untersuchung interindividueller Kompetenzunterschiede im Klassenmanagement von Lehrkräften an. Als eine Wissensfacette des pädagogischen Wissens gilt Klassenmanagement als allgemeines Schlüsselmerkmal der Unterrichtsqualität (Helmke 2015). Der Aufbau von Kompetenzen im Klassenmanagement ist ein Desiderat in allen Phasen der LehrerInnenbildung (Ophardt, Piowar und Thiel 2017). Ein Ansatz zum Kompetenzaufbau im Praxissemester des Lehramtsstudiums wird vorgestellt und evaluiert. Dabei wird untersucht, inwiefern (Selbstwirksamkeits-) Überzeugungen bezüglich inklusiver Bildung den Aufbau und die Anwendung von Klassenmanagementstrategien begünstigen können. 


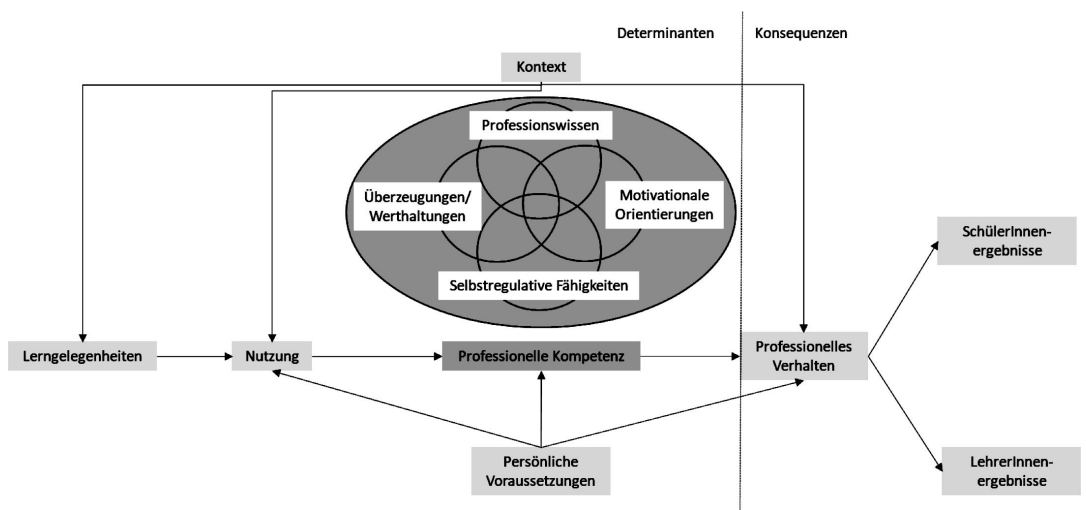

Abbildung 1: Determinanten und Konsequenzen professioneller Kompetenz (nach Kunter, Kleickmann, Klusmann und Richter 2011, 59)

\section{Klassenmanagement}

In einem breiten Begriffsverständnis umfasst Klassenmanagement all jene Lehrkrafthandlungen, die dazu beitragen, eine für das akademische und sozial-emotionale Lernen günstige Umgebung zu schaffen (Evertson und Weinstein 2006). Damit umfasst es neben reaktivem Verhalten der Lehrkraft im Umgang mit Regelverstößen und Unterrichtsstörungen insbesondere die präventiven und proaktiven Lehrkrafthandlungen. Kounin (2006) arbeitet heraus, dass sich erfolgreiche Lehrkräfte nicht wesentlich darin unterscheiden, wie sie auf Unterrichtsstörungen reagieren als vielmehr dadurch, wie sie präventive Strategien zur Vermeidung von Unterrichtsstörungen einsetzen. Im Ergebnis von Befragungen und Unterrichtsbeobachtungen zeigen Wallace, Reschly Anderson, Bartholomay und Hupp (2002), dass Lehrkräfte in Klassen mit positiven akademischen und sozialen Resultaten für SchülerInnen mit und ohne Behinderung weniger als zwei Prozent der Zeit mit der Disziplinierung von SchülerInnen verbringen. 75 Prozent der Zeit sind sie unterrichtend, verwaltend oder interagierend tätig. Sind Lehrkräfte unsicher in der Verwendung präventiver Strategien, verwenden sie häufiger reaktive Strategien (Woodcock und Reupert 2012). In ihrer Meta-Analyse basierend auf 101 zwischen 1967 und 1997 veröffentlichten Studien zum Klassenmanagement stellen Marzano, Marzano und Pickering (2003) vier Komponenten wirksamen Klassenmanagements heraus: (1) Regeln und Prozeduren (d.h. ein bestimmter eingeübter Ablauf beim Verteilen von Arbeitsmaterial), (2) disziplinarische Interventionen, (3) die Lehrkraft-SchülerInnenBeziehung und (4) das » mentale Set « der Lehrkraft (d. h. potenziellen Handlungsbedarf zu identifizieren und umgehend sowie emotional objektiv darauf zu reagieren). 
Die Bedeutung des Klassenmanagements als eine Schlüsselvariable für die Minimierung unangemessenen Verhaltens von SchülerInnen im Unterricht (Oliver, Wehby und Reschly 2011) und die Maximierung der aktiven Lernzeit (Doyle 2006) und damit einer positiven Lernentwicklung der SchülerInnen sind empirisch gut belegt (Brophy 2006; Hattie 2009; Helmke 2015; Wang, Haertel und Walberg 1994). Nicht zuletzt vor dem Hintergrund gestiegener Förder- und Integrationsquoten im Bereich der emotionalen und sozialen Entwicklung (Sekretariat der Ständigen Konferenz der Kultusminister der Länder in der Bundesrepublik Deutschland 2018) dürfte ein effizientes Klassenmanagement zunehmend eine notwendige Bedingung für einen hochwertigen, inklusiven Unterricht darstellen. Lehrkräfte selbst sehen in diesem Bereich und insbesondere zum Berufseinstieg großen Entwicklungsbedarf (Bromfield 2006; Pigge und Marso 1997).

Strittiger als die Umsetzung eines passgenauen Klassenmanagements als notwendige Bedingung für akademisches Lernen im gemeinsamen Unterricht ist die Frage danach, welche Lehr-Lern-Arrangements angehenden Lehrkräften für einen effizienten Aufbau jenes pädagogisch-psychologischen Wissens zur Verfügung gestellt werden müssen. In jüngeren Seminar- und Trainingskonzeptionen zur Professionalisierung von Lehrkräften kommt der Arbeit mit Videovignetten besonderes Augenmerk zu (Seidel und Thiel 2017). Hierbei können Praxisbeispiele in ihrer Komplexität reduziert und Handlungsstrategien unter Einbezug theoretischer Kriterien situationsbezogen identifiziert, reflektiert und ggf. adäquate Alternativen formuliert werden (Micro-teaching, Havers 2015). Eine Verbesserung in der professionellen Wahrnehmung klassenführungsrelevanter Unterrichtsereignisse mittels eines videobasierten Trainings konnten Gold, Förster und Holodynski (2013) nachweisen, wobei die Arbeit mit eigenen gegenüber fremden Unterrichtsvideografien besonders aussichtsreich für den Lernprozess erscheint (Hellermann, Gold und Holodynski 2015). Kleinknecht und Schneider (2013) zeigen auf, dass die Analyse eigener und fremder Unterrichtsvideografien unterschiedliche emotionale, motivationale und kognitive Effekte zur Folge haben kann. Demnach geht die Analyse am fremden Videobeispiel mit einer höheren emotionalen Motivationsbeteiligung und einer intensiveren Analyse problematischer Ereignisse einher.

\section{Überzeugungen von Lehrkräften bezüglich inklusiver Pädagogik}

Berufsbezogene Überzeugungen sind affektiv geprägt und enthalten bewertende Vorstellungen zu Personen (z. B. sich selbst, SchülerInnen und KollegInnen), Lerninhalten und Lernprozessen sowie zu Schule und Gesellschaft (z. B. ihrer Funktion, Standards), welche denk- und handlungsleitend sein können (Reusser und Pauli 2014; Pawelzik 2017). Überschneidungen und Grenzlinien zu Begriffen wie Einstellungen, Haltungen 
etc. können hier keine Erläuterung finden. Nachfolgend werden die von den AutorInnen gebrauchten Begriffe verwendet.

In ihrer Metaanalyse von 26 Studien zeigen de Boer, Pijl und Minnaert (2011), dass Lehrkräfte mehrheitlich neutrale bis negative Einstellungen zur Beschulung von SchülerInnen mit besonderem Unterstützungsbedarf in allgemeinen Grundschulen haben. Hecht, Niedermair und Feyerer (2016) resümieren hingegen eine eher befürwortende Einstellung von PädagogInnen gegenüber inklusiver Bildung. SchülerInnen mit besonderem emotionalen und sozialen Unterstützungsbedarf lösen dabei bei den Lehrkräften signifikant größere Besorgnis aus als SchülerInnen mit anderen Unterstützungsbedarfen (Avramidis, Bayliss und Burden 2000). Sermier Dessemontet, Benoit und Bless (2011) resümieren, dass in den bis dato vorliegenden Forschungsarbeiten bei den Lehrkräften eine positive Einstellung gegenüber der Idee der Integration angenommen wird, sie sich jedoch zurückhaltender dazu äußern, wenn es um die konkrete Umsetzung geht. Einen anderen Aspekt der kognitiven Komponente von Einstellungen hat Sadler (2005) erfasst, indem sie das Wissen von Lehrkräften bezüglich der Unterrichtung von SchülerInnen mit Sprech- und Sprachschwierigkeiten untersuchte. Keine der untersuchten 89 Lehrkräfte erachtete ihr Wissen als ausreichend, 87,6 Prozent stuften es als (sehr) begrenzt ein. Passend dazu empfanden sich diese Lehrkräfte mehrheitlich als (überhaupt) nicht sicher in der Unterrichtung dieser SchülerInnengruppe (affektive Komponente). Die Ergebnisse unterscheiden sich mit dem Unterstützungsbedarf der SchülerInnen. Avramidis und Norwich (2002) schlussfolgern, dass Lehrkräfte eher bereit sind SchülerInnen mit geringen oder physisch/sensorischen Behinderungen inklusiv zu unterrichten als SchülerInnen mit komplexem Unterstützungsbedarf. Lehrkräfte mit weniger Berufsjahren, Erfahrung in inklusiver Bildung und mit sonderpädagogischer Ausbildung haben tendenziell eher eine positive Einstellung zu inklusiver Bildung als Lehrkräfte mit mehr Berufsjahren und ohne entsprechende Erfahrung und Ausbildung (de Boer et al. 2011).

Interessant ist die Frage danach, inwiefern berufsbezogene Überzeugungen von Lehrkräften zu inklusiver Pädagogik mit ihrem pädagogischen Handeln zusammenhängen. Studien belegen, dass die Einstellung von Lehrkräften gegenüber SchülerInnen mit schwierigem Verhalten einen Einfluss auf die Wahl der Intervention hat (Ghaith und Yaghi 1997; Milner 2002). Jordan, Schwartz und McGhie-Richmond (2009) zeigen, dass Lehrkräfte mit einer positiveren Haltung gegenüber SchülerInnen mit besonderem Unterstützungsbedarf und der Überzeugung, dass sie für alle SchülerInnen verantwortlich sind, tendenziell wirksamere Unterrichtspraktiken für alle SchülerInnen der Lerngruppe umsetzen als Lehrkräfte mit einer negativeren Haltung und weniger Verantwortungsgefühl. Um zu überprüfen, ob sich affektive Lehrkraftmerkmale in ihrem pädagogischen Handeln wiederfinden, führten Sharma und Sokal (2016) neben Befragungen auch Unterrichtsbeobachtungen durch. Wenngleich die Ergebnisse aufgrund der Stichprobengröße- und gewinnung $(n=5)$ Limitationen unterliegen, weisen 
die Daten in die erwartete Richtung, das heißt, dass Lehrkräfte mit einer positiveren Einstellung und weniger Bedenken bezüglich inklusiver Pädagogik wirksamer in der Umsetzung inklusiver Unterrichtspraxis sind.

\section{Selbstwirksamkeitsüberzeugungen von Lehrkräften bezüglich ihrer inklusiven Unterrichtspraxis}

Selbstwirksamkeitsüberzeugungen werden nach Bandura (1977) verstanden als subjektive Einschätzung der eigenen Fähigkeiten, angestrebte Handlungen realisieren zu können. Jene Einschätzung trägt zur Handlungsregulierung bei, indem Aufgaben gemäß ihrer Angemessenheit (z. B. hinsichtlich des Schwierigkeitsgrades) ausgewählt werden (Schwarzer und Warner 2014).

Der Zusammenhang von Selbstwirksamkeitsüberzeugungen von Lehrkräften und Aspekten der Unterrichtsvorbereitung und -durchführung sind empirisch gut belegt (Gebauer 2013). Beispielswiese sind Lehrkräfte mit ausgeprägteren Selbstwirksamkeitsüberzeugungen offener für neue Strategien (Fuchs, Fuchs und Bishop 1992) und besser in der Anwendung von Klassenmanagementstrategien (Woolfolk, Roseloff und Hoy 1990). Baker (2005) ermittelte eine starke Korrelation $r=0,56, p<0,001$ zwischen der Selbstwirksamkeitsüberzeugung von Lehrkräften im Klassenmanagement und der Bereitschaft, der Fähigkeit und dem Willen für den Umgang mit herausforderndem Verhalten. Der Aufbau inklusiver Selbstwirksamkeitsüberzeugungen kann durch inklusionsbezogene Studieninhalte (Kopp 2009), entsprechende Verhaltensvorbilder oder positiv besetzte Erfahrungen (Schwarzer und Warner 2014) begünstigt werden.

Hecht et al. (2016) arbeiteten heraus, dass PädagogInnen tendenziell über eher hohe inklusive Selbstwirksamkeitsüberzeugungen verfügen und dass diese ihrerseits (konkret bezüglich des Umgangs mit schwierigem Verhalten und der Zusammenarbeit in einem professionellen Team) dazu beitragen können, inklusionsbezogene Überzeugungen vorherzusagen. Sermier Dessemontet et al. (2011) gehen zudem davon aus, dass der Aufbau inklusionsbezogener Selbstwirksamkeitsüberzeugungen inklusionsoffene Überzeugungen begünstigen kann. Und wenn diese wiederum, wie oben aufgezeigt, die Realisierung wirksamer Unterrichtspraktiken begünstigen können, birgt das ein nutzbar zu machendes Potenzial für einen zielgerichteten Aufbau professioneller Handlungskompetenz.

\section{Seminarkonzept}

Klassenmanagement als unseres Erachtens notwendige Bedingung inklusiver Unterrichtspraxis umfasst eine Verbindung aus deklarativem Wissen und praktischem 
Können und kann nur im unterrichtlichen Handeln aufgebaut werden (Blüthmann, Ophardt, Thiel und Felsberger 2011). Insofern wurden Module zum Klassenmanagement in Begleitseminaren zum Praxissemester im Masterstudium Lehramt platziert, welches an der Universität Potsdam eine viermonatige schulische Praxisphase miteinschließt. In diesem Zeitraum sind Studierende vier Tage pro Woche in der Schule, um dort zu hospitieren (66 Schulstunden) und selbstständig zu unterrichten (50 Schulstunden). An einem Tag in der Woche nehmen die Studierenden an universitären Begleitveranstaltungen der Bildungswissenschaft und ihren jeweiligen zwei Fachdidaktiken teil. Neben einer Vor- und Nachbereitungsveranstaltung stehen jeweils fünf Seminare im Umfang von zweieinhalb Stunden für den Austausch zu unterrichtsbezogenen Fragestellungen zur Verfügung. Inhalte zum Klassenmanagement wurden in einzelnen Blöcken des bildungswissenschaftlichen Begleitseminars eingebettet. Zur Überbrückung der Kluft zwischen universitärer Lehre und pädagogischer Praxis sowie anknüpfend an die Forschung zum Microteaching und aussichtsreichen Befunden zu den Effekten videogestützten Trainings hinsichtlich des Aufbaus von Professionswissen wurde ein Seminarkonzept zum Klassenmanagement mit videogestütztem Micro-teaching entwickelt. Nach Klinzing (2002) umfasst es im Kern vier Elemente: Vermittlung theoretischen Hintergrundwissens, Methoden zur kognitiven Aneignung spezifischen Verhaltens, praktische Übungen in experimentellen Settings und Feedback. Methodisch zeichnet sich das Seminarkonzept durch die kriteriengeleitete Reflexion eigener und fremder Unterrichtsvignetten aus. Zur Systematisierung dieses Vorgehens wurden die Reflexionsschritte Beschreiben, Erklären, Handlungsmöglichkeiten generieren und Konsequenzen ableiten eingeführt (Lohse-Bossenz und Krauskopf 2017), welche eine Adaption des reflexiven Dreischritts Beschreiben, Erklären und Integrieren darstellen. Für die kriteriengeleitete Reflexion wurde zudem auf Basis eines umfangreichen Literaturreviews ein heuristisches Modell zum Klassenmanagement erarbeitet (vgl. Abbildung 2). Es umfasst die drei Dimensionen Allgegenwärtigkeit, Prozessmanagement und Verhaltensmanagement mit jeweils drei Facetten (zur empirischen Überprüfung der faktoriellen Struktur vgl. Gehrmann, Karpyuk, linka, Gerlach, Lohse-Bossenz und Schramm in Vorbereitung). Allgegenwärtigkeit beinhaltet die (non-)verbale wie physische Präsenz, Monitoring des SchülerInnenverhaltens sowie die Verarbeitung und Umsetzung simultan ablaufender Handlungen (Multitasking). Prozessmanagement subsummiert Lehrkrafthandlungen zur Strukturierung und Steuerung von Unterricht, Zeit- und Raummanagement. In der Dimension Verhaltensmanagement finden sich unter dem Aspekt Verhaltenserwartungen vornehmlich präventive und beim Umgang mit Regelverstößen und Unterrichtsstörungen eher reaktive Strategien. Beziehungsmanagement bezieht sich auf die Ausgestaltung der Lehrkraft-SchülerInnenbeziehung. 

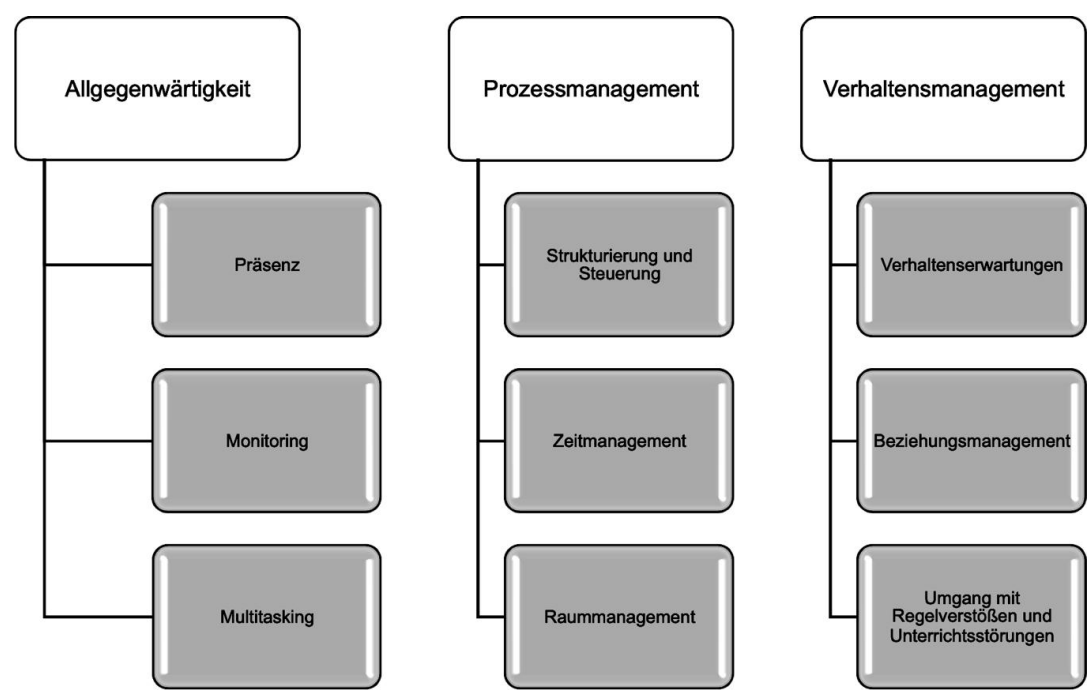

Abbildung 2: Modell zum Klassenmanagement (nach Linka und Gehrmann 2018, 203)

Nach einem Theorieinput zum Klassenmanagement, Übungen zur systematischen, kriteriengeleiteten Reflexion von klassenmanagementbezogenem Lehrkrafthandeln sowie Informationen über organisatorische und technische Anforderungen einer Unterrichtsvideografie wurden die Studierenden dazu aufgefordert, ihren eigenverantwortlich durchgeführten Unterricht zu videografieren und dem Seminar klassenmanagementrelevante Auszüge bereitzustellen, um diese gemeinsam anhand der Kriterien zu reflektieren. Vor dem Hintergrund eines umfangreichen Genehmigungsverfahrens zur Aufnahme von Unterrichtsvideos konnten jedoch nicht alle Studierenden eine Unterrichtsvideografie anfertigen. Alternativ fertigten sie Textvignetten an.

\section{Hypothesen}

Im Folgenden werden Hypothesen zu den Effekten der klassenmanagementbezogenen Intervention in Form der beschriebenen Seminarkonzeption sowie dem Erklärungsgehalt von interindividuellen Unterschieden in den Überzeugungen und Selbstwirksamkeitsüberzeugungen bezüglich inklusiver Bildung formuliert.

\section{$>$ Performanzen im Klassenmanagement}

$\mathrm{H}_{1 \mathrm{a}}$ : Die Studierenden der Interventionsgruppe (IG) und der Vergleichsgruppe (VG) (Gesamtstichprobe) schneiden im Post-Test hinsichtlich ihrer Per- 
formanzen im Klassenmanagement signifikant höher ab als im Prä-Test. (Praxissemester als Lerngelegenheit)

$\mathrm{H}_{1 \mathrm{~b}}$ : Die Selbsteinschätzungen der Studierenden zu ihren Performanzen im Klassenmanagement fallen im Post-Test gegenüber dem Prä-Test in der IG signifikant höher aus als in der VG (Interventionseffekt).

$\mathrm{H}_{1 \mathrm{c}}$ : Der Interventionseffekt fällt bei Studierenden signifikant stärker aus, die eine eigene Unterrichtsvideografie zur gemeinsamen Reflexion bereitgestellt haben als bei Studierenden, die eine Textvignette angefertigt haben.

> Überzeugungen zu inklusiver Pädagogik

$\mathrm{H}_{2 \mathrm{a}}$ : Die Studierenden der IG und der VG (Gesamtstichprobe) schneiden im Post-Test hinsichtlich ihrer Überzeugungen zu inklusiver Pädagogik signifikant positiver ab als im Prä-Test. (Praxissemester als Lerngelegenheit)

$\mathrm{H}_{2 \mathrm{~b}}$ : Je positiver die Überzeugungen zu inklusiver Pädagogik, umso ausgeprägter das Klassenmanagement.

> Selbstwirksamkeitsüberzeugungenzur Umsetzungeinerinklusiven Unterrichtspraxis $\mathrm{H}_{3 \mathrm{a}}$ : Die Studierenden der IG und der VG (Gesamtstichprobe) schneiden im Post-Test hinsichtlich ihrer Selbstwirksamkeitsüberzeugungen zur Umsetzung einer inklusiven Unterrichtspraxis signifikant höher ab als im PräTest. (Praxissemester als Lerngelegenheit)

$\mathrm{H}_{3 \mathrm{~b}}$ : Je ausgeprägter die Selbstwirksamkeitsüberzeugungen bezüglich der Umsetzung einer inklusiven Unterrichtspraxis, umso ausgeprägter das Klassenmanagement.

Zudem wird explorativ untersucht, welche Bedeutung die Überzeugungen und Selbstwirksamkeitsüberzeugungen bezüglich inklusiver Bildung der Studierenden für den Interventionseffekt haben.

\section{Methode}

\section{Stichprobe}

Die Durchführung und Evaluation des Seminars erfolgte im SoSe 2017 und WiSe 2017/18 in Form eines quasi- experimentellen Prä-Post-Kontrollgruppendesigns. Zwei parallel stattfindende Begleitseminargruppen der Bildungswissenschaften stellten die Interventions- und die Vergleichsgruppe (IG, VG). Die Seminargruppen wurden nach studienfachorganisatorischen Kriterien vom Zentrum für Lehrerbildung und Bildungsforschung der Universität Potsdam zusammengestellt (nicht randomisiert). Anschließend bildete eine komplette Seminargruppe die IG und eine andere die 
VG. In beiden Semestern haben insgesamt 36 Studierende an einem videogestützten Begleitseminar mit dem Fokus auf das Klassenmanagement teilgenommen (IG). Bei Berücksichtigung der vollständigen Datensätze werden nachfolgend die Angaben von 35 Studierenden in der IG und 34 Studierenden in der VG präsentiert. Die Studierenden beider Gruppen waren überwiegend weiblich, der angestrebte Abschluss war zumeist das Lehramt der Primarstufe (z. T. mit Schwerpunkt Inklusion; vgl. Tabelle 1).

\begin{tabular}{lllll}
\hline & SoSe $\mathbf{2 0 1 7}$ & \multicolumn{3}{c}{ WiSe 2017/18 } \\
& IG & VG & IG & VG \\
\multirow{2}{*}{ Geschlecht } & 18 weiblich & 19 weiblich & 13 weiblich & 13 weiblich \\
& 1 männlich & 1 männlich & 3 männlich & 1 männlich \\
\multirow{2}{*}{ Studiengang } & 11 LA Primar & 18 LA Primar Ink & 12 LA Primar & 9 LA Primar \\
& 8 LA Sek I\& II & 2 LA Primar & 4 LA Primar Ink & 5 LA Primar Ink \\
\hline
\end{tabular}

Tabelle 1: Stichprobe (Anmerkung: LA Primar = Lehramt für die Primarstufe; LA Primar Ink = Lehramt für die Primarstufe mit dem Schwerpunkt Inklusionspädagogik; LA Sek I \& II = Lehramt für die Sekundarstufen I und II)

\section{Instrumente}

Zur Erfassung von Veränderungen in den klassenmanagementbezogenen Performanzen wurde die Lehrkräfteversion des Instruments zur $\underline{m}$ ultiperspektivischen $\underline{E}$ fassung des $\underline{K}$ lassenmanagements von $\underline{L}$ ehrkräften $\gg$ MERKMAL-L « genutzt (Gehrmann et al. in Vorbereitung). Dieses entspricht in seinen Dimensionen und Facetten dem Modell in Abbildung 2. Von den insgesamt 32 Items erfassen neun Items Aspekte der Allgegenwärtigkeit (z. B. »Ich bemerke umgehend, wenn sich jemand mit etwas anderem beschäftigt.«), elf Items betreffen das Prozessmanagement (z. B. »Ich nutze Gesten und spezifische Signale zur Steuerung von SchülerInnenhandlungen.«) und zwölf Items das Verhaltensmanagement der Lehrkräfte (z. B. »Ich verweise bei Regelverstößen und Unterrichtsstörungen auf vereinbarte Regeln.«). Die Selbsteinschätzung zielt auf Performanzen im Klassenmanagement und erfolgt auf einer sechsstufigen Likert-Skala von eins »Trifft gar nicht $\mathrm{zu} \ll$ bis sechs »Trifft völlig zu «. Die Annahme, dass sich Kompetenz in Performanz zeigt, lehnt sich an Abbildung 1 sowie das Modell von Blömeke, Gustafsson und Shavelson (2015) an, wonach sich, vermittelt über situationsbezogene Fähigkeiten der Wahrnehmung, Interpretation und Entscheidungsfindung, Kompetenz in Performanz transformiert.

Einstellungen, Haltungen und Bedenken zu inklusiver Pädagogik wurden mittels der Skala The Sentiments, Attitudes and Concerns about Inclusive Education Revised 
(SACIE-R; Forlin, Earle, Loreman und Sharma 2011) in der deutschen Übersetzung nach Feyerer et al. (2016) erhoben. Vor dem Hintergrund akzeptabler interner Konsistenzen der Originalskalen (Cronbach Alpha zwischen 0,62 und 0,80) wurde die dreifaktorielle Struktur übernommen (Feyerer 2014). Jeweils fünf Items betreffen die negativen Einstellungen bezüglich Beeinträchtigung (z. B. »Ich scheue mich davor, einer Person mit Beeinträchtigungen in die Augen zu schauen. «), fünf Items die positiven Haltungen zur inklusiven Schule (z.B. »Auch SchülerInnen, die unkonzentriert sind, sollten gemeinsam mit allen anderen unterrichtet werden. «) und fünf Items die Bedenken bezüglich der Umsetzung inklusiven Unterrichts der Lehrkräfte (z. B. »Ich habe die Sorge, dass ich nicht über die erforderlichen Kenntnisse und Fähigkeiten verfüge, um SchülerInnen mit Beeinträchtigungen zu unterrichten.«). Die Antwortskala reicht jeweils von eins $\gg$ Stimmt überhaupt nicht « bis vier $»$ Stimmt völlig $«$. Nach der Umkodierung stehen hohe Werte für eine positive Einstellung und geringe für Bedenken bezüglich inklusiver Bildung.

Zur Erfassung der Selbstwirksamkeitsüberzeugungen von Lehrkräften bezüglich ihrer Umsetzung inklusiver Unterrichtspraxis wurde die Teacher Efficacy for Inclusive Practices Scale (TEIP; Sharma, Loreman und Forlin 2012) in der deutschen Übersetzung nach Feyerer et al. (2016) verwendet. Aufgrund hoher interner Konsistenzen (Cronbach Alpha zwischen 0,85 und 0,93) wurde die dreifaktorielle Struktur beibehalten (Sharma et al. 2012). Die 18 Items verteilen sich zugleich auf die drei Subskalen zur individualisierenden Unterrichtsgestaltung (z. B. »Ich kann genau abschätzen, was SchülerInnen von dem, was ich gelehrt habe, verstanden haben.«), interdisziplinärer Kooperation (z. B. »Ich kann Familien unterstützen, ihren Kindern zu helfen, in der Schule gut voranzukommen.«) und dem wirksamen Umgang mit störendem Verhalten (z.B. »Ich kann den SchülerInnen meine Erwartungen an ihr Verhalten klar vermitteln. «) jeweils mit den Antwortskalen von eins »Trifft überhaupt nicht $\mathrm{zu} \ll$ bis sechs $\gg$ Trifft voll zu«.

\section{Auswertung}

Zuerst wurde mittels t-Test für unabhängige Stichproben kontrolliert, ob die IG und die VG bezüglich ihrer Performanzen im Klassenmanagement sowie ihren Überzeugungen und Selbstwirksamkeitsüberzeugungen bezüglich inklusiver Bildung vor der Intervention vergleichbar sind (Prä-Test). Die dafür notwendige Bedingung der Varianzhomogenität wurde jeweils mittels Levene-Test überprüft. Zur Einstufung der praktischen Bedeutsamkeit statistisch signifikanter Ergebnisse wird jeweils die Effektstärke $(d)$ von Cohen (1988) angegeben. Werte $\mathrm{ab} d_{\text {Cohen }}=0,2$ bis 0,4 gelten als kleine Effekte, $d_{\text {Cohen }}=0,5$ bis 0,7 als mittlere Effekte und $d_{\text {Cohen }}>0,8$ als große Effekte. 
Zur Überprüfung der Hypothesen $\mathrm{H}_{1 \mathrm{a}}, \mathrm{H}_{2 \mathrm{a}}, \mathrm{H}_{3 \mathrm{a}}$ wonach sich bei allen Studierenden im Praxissemester signifikante Zuwächse in den aufgeführten drei Bereichen finden, wurde ein Vergleich der Mittelwerte vom Prä- und Post-Test mittels t-Test für abhängige Stichproben durchgeführt und bei Hinweisen auf signifikante Zuwächse die Effektstärke $d_{\text {repeated Measures }}$ berechnet (Interpretationskonvention s. o.).

Der Interventionseffekt $\left(\mathrm{H}_{1 \mathrm{~b}}\right)$ und der Effekt des Einsatzes von Video- und Textvignetten $\left(\mathrm{H}_{\mathrm{lc}}\right)$ wurde mittels Varianzanalyse mit Messwiederholungs- und Gruppenfaktor ermittelt (Mixed ANOVA/rm ANOVA). Dabei ist der Messwiederholungsfaktor jeweils die Performanz im Klassenmanagement (Prä und Post, Inner-Subjekt-Faktor). Der Gruppenfaktor ist bei $\mathrm{H}_{1 \mathrm{~b}}$ die Zugehörigkeit zur IG oder VG und bei $\mathrm{H}_{1 \mathrm{c}}$ die Verwendung von Video- oder Textvignetten (Zwischen-Subjekt-Faktor). Im SoSe 2017 führten acht von 19 Studierenden eine Unterrichtsvideografie durch, im WiSe alle 16 Studierenden. Nach Angaben der elf Studierenden im SoSe 17, die keine Unterrichtsvideografie durchführten, fehlte das Einverständnis der Eltern in sechs Fällen, der Schulleitung in fünf Fällen und der betreuenden Lehrkraft in drei Fällen (Mehrfachnennungen waren möglich). Als Effektmaß wird hier das partielle Eta-Quadrat $\left(\eta_{p}^{2}\right)$ angegeben. Nach Cohen (1988) liegen die Grenzen des Effektes bei 0,01 (kleiner Effekt), 0,06 (mittlerer Effekt) und 0,14 (großer Effekt).

Die Ergebnisse zu den Zusammenhangshypothesen $\mathrm{H}_{2 \mathrm{~b}}$ und $\mathrm{H}_{3 \mathrm{~b}}$ basieren auf bivariaten Korrelationsanalysen. Cohen (1988) gibt für die Interpretation von Spearmans Korrelationskoeffizient $\left(r_{s}\right)$ die folgenden Intervalle an: $r_{s}=0,1$ bis 0,3 (kleiner Effekt), $r_{s}=0,3$ bis 0,5 (mittlerer Effekt), $r_{s}>0,5$ (starker Effekt). Für die Explorative Analyse zur Bedeutsamkeit von Überzeugungen und Selbstwirksamkeitsüberzeugungen bezüglich inklusiver Bildung zu Beginn des Praxissemesters für den Interventionseffekt wurden anhand der über/unter dem Mittelwert liegenden Ausprägungen in den Überzeugungen und Selbstwirksamkeitsüberzeugungen vier Gruppen gebildet und ihre Mittelwerte (Post-Prä) verglichen sowie die Effektstärke $d_{\text {repeated Measures }}$ berechnet.

\section{Ergebnisse}

\section{Performanzen im Klassenmanagement}

Bereits im Prä-Test beider Semester schätzten die Studierenden der IG und der VG ihre Performanzen im Klassenmanagement positiv ein. Auf der sechsstufigen Skala lag die Selbsteinschätzung im Mittel zwischen $M=4,22(S D=0,87)$ und $M=4,86$ $(S D=0,42)$. In allen Semestern und Gruppen beurteilten sich die Studierenden hinsichtlich des Aspekts Allgegenwärtigkeit (Präsenz, Monitoring, Multitasking) relativ 
am niedrigsten $(M=3,46(S D=0,50)$ bis $M=4,48,(S D=0,52))$ und mit Ausnahme der IG im WiSe 2017/18 bezüglich des Verhaltensmanagements (Verhaltenserwartungen, Beziehungsmanagement, Umgang mit Regelverstößen und Unterrichtsstörungen) am höchsten $(M=4,29(S D=0,91)$ bis $M=5,15(S D=0,41))$. Die selbst eingeschätzte Performanz im Prozessmanagement (Strukturierung und Steuerung, Raumund Zeitmanagement) lag zwischen $M=4,36(S D=0,91)$ und $M=4,85(S D=0,46)$. Ein statistisch bedeutsamer Unterschied zwischen der IG und der VG zeigt sich im Bereich des Klassenmanagements einzig im SoSe 2017 bezüglich des Aspektes Verhaltensmanagement $(t(37)=-3,166, p=0,003)$. Der Effekt kann als mittelstark eingestuft werden $\left(d_{\text {Coben }}=0,64\right)$. Insgesamt können die beiden Gruppen angesichts ihrer Selbsteinschätzungen zu den Performanzen im Klassenmanagement zum Prä-Test jedoch als vergleichbar angesehen werden.

Erwartungsgemäß nehmen die klassenmanagementbezogenen Performanzen bei allen Studierenden im Praxissemester signifikant zu $\left(\mathrm{H}_{1 \mathrm{a}}, t(68)=-4,738, p=0,000, d_{\text {repeated }}\right.$ Measures $=0,50)$. Die Effekte für die Teilbereiche rangieren dabei auf ähnlichem Niveau: Allgegenwärtigkeit $d_{\text {repeated Measures }}=0,46$, Prozessmanagement $d_{\text {repeated Measures }}=0,37$, Verhaltensmanagement $d_{\text {repeated Measures }}=0,40$.

Es stellt sich die Frage, ob dieser Zuwachs auf die Intervention (konkret: das Seminarkonzept) zurückgeführt werden kann. Die Voraussetzungen für die Durchführung einer mixed ANOVA liegen vor: Da der Inner-Subjekt-Faktor nur zwei Stufen hat, ist die Bedingung der Sphärizität erfüllt. Auch Homoskedastizität ist gemäß dem Levene-Test für alle Variablen gegeben $(p>0,05)$. Im Ergebnis der Analyse liegt kein signifikanter Interaktionseffekt vor, wonach sich die Performanzen im Klassenmanagement über alle Dimensionen hinweg in Abhängigkeit der Intervention unterscheiden würden. Die Veränderungen in den Performanzen im Klassenmanagement sind vor dem Hintergrund der Analysen einzig auf den Faktor Zeit $(F(1,67)=22,49$, $\left.\mathrm{p}<0,001, \eta_{\mathrm{p}}{ }^{2}=0,25\right)$ und nicht auf die Intervention zurückzuführen. Ein signifikanter Interaktionseffekt zeigt sich einzig hinsichtlich der Dimension Verhaltensmanagement $\left(F(1,67)=6,09, p=0,02, \eta_{p}{ }^{2}=0,08\right)$, welcher wesentlich auf das SoSe 2017 zurückzuführen ist $\left(F(1,37)=6,27, p=0,02, \eta_{p}^{2}=0,15\right)$ (vgl. Linka und Gehrmann 2018). Der Effekt der Intervention für die Performanzen im Verhaltensmanagement ist als mittel bzw. groß zu bewerten. Die $\mathrm{H}_{1 \mathrm{~b}}$ kann insgesamt nicht oder nur in Teilen bestätigt werden.

Im Rahmen einer tabletbasierten Befragung am Ende des Praxissemesters wurden die 24 Studierenden der IGs, die eine Unterrichtsvideografie durchführten, danach gefragt, warum sie dies taten (Mehrfachnennungen möglich). Mit einer Ausnahme führten die Studierenden eine Unterrichtsvideografie aufgrund der Seminarvorgaben durch, nur jede/r Vierte, weil er/sie sich davon einen Erkenntnisgewinn versprach. Eine Person gab an, dass sie sich davon keinen Erkenntnisgewinn versprochen habe. 
Niemand gab an, dass es zu aufwendig, verunsichernd oder ihnen unangenehm gewesen wäre. Weiterhin gaben die 69 Studierenden dieser Stichprobe auf einer elfstufigen Skala $(0=»$ Gar nicht $\ll, 10=»$ Sehr intensiv «) an, dass sie die Reflexion eigener Unterrichtsvideografien als besonders lernwirksam erachten $(M=8,19(S D=1,82))$. Auch die Arbeit mit fremden Unterrichtsvideografien $(M=6,58(S D=1,94))$ und eigenen Textvignetten $(M=5,78(S D=2,46))$ erscheint ihnen förderlich für den Erkenntniszuwachs. Fremde Textvignetten $(M=4,72(S D=2,33))$ nehmen in diesem Zusammenhang eine nachgeordnete Position ein (ebd.).

Zwar deuten auch die Mittelwertvergleiche in die erwartete Richtung, dass Studierende mehr vom Seminarkonzept profitieren, wenn sie Video- statt Textvignetten verwenden, jedoch kann kein signifikanter Interaktionseffekt festgestellt werden, wonach sich die Performanzen im Klassenmanagement in Abhängigkeit von der Vignettenform unterscheiden. Eine abschließende Beantwortung der $\mathrm{H}_{1 \mathrm{c}}$ muss offen bleiben.

\section{Überzeugungen zu inklusiver Pädagogik}

Nach den Ergebnissen der Befragung mit den vierstufigen Antwortskalen zu ihren Einstellungen, Haltungen und Bedenken zu inklusiver Pädagogik haben die Studierenden im Mittel kaum negative Einstellungen gegenüber Beeinträchtigung $(M=2,84(S D=0,47)$ bis $M=3,30(S D=0,54))$, recht positive Haltungen zur inklusiven Schule $(M=2,73(S D=0,56)$ bis $M=3,21(S D=0,42))$ und auf mittlerem Niveau ausgeprägte Bedenken bezüglich der Umsetzung inklusiven Unterrichts $(M=1,63(S D=0,39)$ bis $M=2,26(S D=0,45))$. Im SoSe 2017 haben die Studierenden der VG signifikant weniger negative Einstellungen $(t(37)=-2,347$, $p=0,024)$ und positivere Haltungen $(t(37)=-2,795, p=0,008)$ als die Studierenden der IG. Die Effekte sind als mittel bis groß zu bewerten (Einstellungen $d_{\text {Coben }}=0,75$, Haltungen $\left.d_{\text {Cohen }}=0,90\right)$ und wahrscheinlich darauf zurückzuführen, dass 18 von 20 Studierende der VG aufgrund ihrer Schwerpunktsetzung Inklusionspädagogik entsprechende universitäre Lerninhalte und schulische Lerngelegenheiten erhalten haben (vgl. Stichprobe). Signifikante Unterschiede bezüglich der Bedenken zu inklusiver Pädagogik zeigen sich hier nicht, jedoch im WiSe 2017/18. Dort haben die Studierenden der IG weniger Bedenken als Studierende der VG $(t(28)=2,436$, $p=0,021$; starker Effekt $\left.d_{\text {Cohen }}=0,89\right)$. Damit hat im SoSe 2017 die VG und im WiSe 2017/18 die IG positivere Ausprägungen in ihren Überzeugungen zu inklusiver Pädagogik.

Die Veränderungen in den hier erfassten Überzeugungen zu inklusiver Pädagogik sind für die Gesamtstichprobe im Prä-Post-Verlauf nicht statistisch bedeutsam 
$(t(68)=1,101, p=0,275) . \mathrm{H}_{2 \mathrm{a}}$ kann damit nicht bestätigt werden. Auch die $\mathrm{H}_{2 \mathrm{~b}}$, wonach Überzeugungen zu inklusiver Pädagogik positiv mit Performanzen im Klassenmanagement korrelieren, kann weder für die Teil- noch für die Gesamtskala bestätigt werden.

\section{Selbstwirksamkeitsüberzeugungen zur Umsetzung einer inklusiven Unterrichtspraxis}

Auch hinsichtlich der Selbstwirksamkeitsüberzeugungen zur Umsetzung einer inklusiven Unterrichtspraxis deuten die Angaben der Studierenden auf der sechsstufigen Antwortskala mit dem Fokus auf eine individualisierende Unterrichtsgestaltung $(M=4,10(S D=0,66)$ bis $M=4,60(S D=0,66))$, interdisziplinäre Kooperation $(M=3,97(S D=0,69)$ bis $M=4,55(S D=0,97))$ und den Umgang mit störendem Verhalten $(M=3,92(S D=0,73)$ bis $M=4,48(S D=0,51))$ in eine deutlich positive Richtung. Statistisch bedeutsame Unterschiede zwischen der IG und der VG zeigen sich ausschließlich im SoSe 2017, dabei jedoch bei allen drei Skalen (individualisierende Unterrichtsgestaltung $t(37)=-2,326, p=0,026, d_{\text {Cohen }}=0,75$; interdisziplinäre Kooperation $t(37)=-2,163, p=0,037, d_{\text {Cohen }}=0,69$; Umgang mit störendem Verhalten $\left.t(37)=-2,748, p=0,009, d_{\text {Coben }}=0,88\right)$. Im SoSe 2017 empfinden sich die Studierenden der VG als wirksamer bei der Umsetzung einer inklusiven Unterrichtspraxis als die Studierenden der IG. Dieser Effekt könnte erneut auf die überwiegende Studienschwerpunktsetzung Inklusionspädagogik in der VG zurückzuführen sein.

Die Veränderungen in den hier erfassten Selbstwirksamkeitsüberzeugungen zur Umsetzung inklusiver Unterrichtspraxis sind für die Gesamtstichprobe im Prä-PostVerlauf statistisch bedeutsam $\left(\mathrm{H}_{3 \mathrm{a}}, t(68)=-4,388, p=0,000, d_{\text {repeated Measures }}=0,49\right)$. Die Effektstärke liegt hinsichtlich individualisierender Unterrichtsgestaltung bei $d_{\text {repeated Measures }}=0,33$ (kleiner Effekt), hinsichtlich interdisziplinärer Kooperation bei

$d_{\text {repeated Measures }}=0,25$ (kleiner Effekt) und hinsichtlich des Umgangs mit Störungen bei $d_{\text {repeated Measures }}=0,62$ (mittlerer Effekt). Damit profitieren die Studierenden am Ende des Praxissemesters insbesondere bezüglich der selbst wahrgenommenen Wirksamkeit im Umgang mit herausforderndem SchülerInnenverhalten. $\mathrm{H}_{3 \mathrm{a}}$ kann bestätigt werden.

Die Korrelation der Prä-Post-Differenzen deutet darauf hin, dass sich mit zunehmenden Selbstwirksamkeitsüberzeugungen auch die Performanzen im Klassenmanagement erhöhen $\left(r_{s}=0,37, p<0,01\right)$. Dieser Zusammenhang zeigt sich insbesondere zwischen der Skala zum wirksamen Umgang mit Unterrichtsstörungen und allen Teilskalen des Klassenmanagements $\left(r_{s}=0,33\right.$ bis $\left.r_{s}=0,45, p<0,01\right)$. 


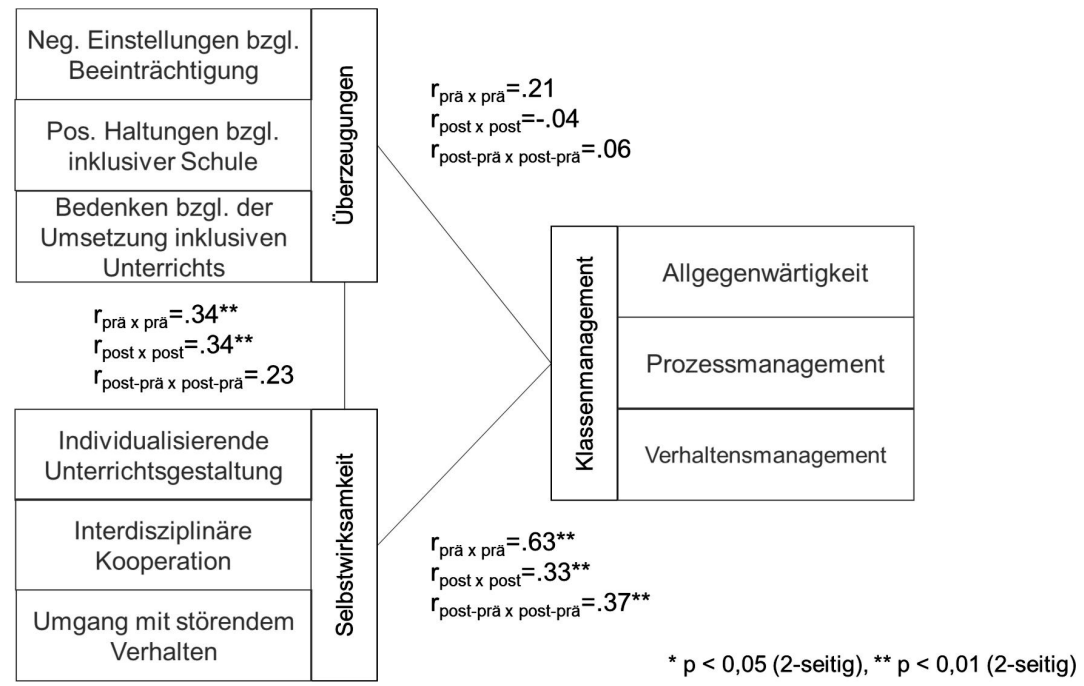

Abbildung 3: Korrelationen

\section{Die Bedeutung von (Selbstwirksamkeits-) Überzeugungen bezüglich inklusiver Bildung für den Interventionseffekt}

Nachfolgend wird der Frage nachgegangen, ob Studierende mit positiven/negativen Überzeugungen (Ü+/Ü-) und Studierende mit niedrigen/hohen Selbstwirksamkeitsüberzeugungen $(S W+/ S W-)$ bezüglich inklusiver Bildung in besonderer Weise vom Seminarkonzept profitierten. Die geringsten Veränderungen in ihren klassenmanagementbezogenen Performanzen zeigen sich bei den Studierenden, die zu Beginn des Praxissemesters in Relation zur Referenzgruppe weniger überzeugt von den Zielen inklusiver Bildung waren. Die Angaben zu den Performanzen im Klassenmanagement (Post-Prä) fallen für jene mit relativ niedrigeren $\left(n_{\ddot{U} / S W-}=7\right.$, $M=0,29(S D=0,49), t(6)=-1,555, p=0,171)$ und relativ höheren Selbstwirksamkeitsüberzeugungen zur Umsetzung inklusiven Unterrichts vergleichbar stabil aus $\left(n_{U-/ S W_{+}}=9, M=0,29(S D=0,50), t(8)=-1,732, p=0,121\right)$. Sind Studierende jedoch überzeugter von den Zielen inklusiver Bildung nehmen ihre klassenmanagementbezogenen Handlungen eher zu $\left(n_{\ddot{U}+S W_{-}}=6, M=0,63(S D=0,27), t(5)=-5,767\right.$, $\left.p=0,002 / n_{\ddot{U}+S W+}=13, M=0,48(S D=0,90), t(12)=-1,905, p=0,081\right)$. Das ist insbesondere dann der Fall, wenn sie sich zu Beginn des Praxissemesters weniger wirksam in der Umsetzung inklusiver Unterrichtspraxis einschätzten $\left(d_{\text {repeated Measures }}\right.$ $\ddot{U}+/ S W=3,04)$. 


\section{Diskussion}

Ziel des vorliegenden Beitrags ist die Evaluation eines Seminarkonzeptes, welches auf den Aufbau von Kompetenzen im Klassenmanagement angehender Lehrkräfte zielt. Zusammenhänge zwischen diesem Teil des Professionswissens und berufsbezogenen Überzeugungen und Selbstwirksamkeitsüberzeugungen zu inklusiver Bildung wurden analysiert.

Zwar deuten die Befunde in die erwartete Richtung einer Zunahme von klassenmanagementbezogenem Handeln in der IG, jedoch erscheint dieser Zuwachs in Relation mit der VG insgesamt statistisch nicht oder bezüglich des Verhaltensmanagements wenig bedeutsam. Dies könnte neben einem grundsätzlichen Optimierungsbedarf der Intervention mehrere Ursachen haben: Beispielsweise könnte die Intervention nicht konsequent genug umgesetzt worden sein. Kramer, König, Kaiser, Ligtvoet und Blömeke (2017) arbeiteten heraus, dass sich ein systematischer Aufbau von Wissen im Klassenmanagement im Zeitverlauf eines Semesters nur dann feststellen lässt, wenn Studierende ein spezifisch auf das Klassenmanagement ausgerichtetes Seminar besuchen. Wenngleich die Platzierung in Begleitseminaren des Praxissemesters günstig erschien, so blieb zugunsten der ganzheitlich orientierten Perspektive auf die pädagogischen Erfahrungen der Studierenden insgesamt doch nur ein Teil der Zeit in den fünf Blöcken à zweieinhalb Stunden für die kriteriengeleitete Analyse des Klassenmanagements. Die Einschätzung der Intensität der Auseinandersetzung mit dem Thema Klassenmanagement lag bei den Studierenden der IG auf einer elfstufigen Skala im universitären Bereich bei $M=6,06$ $(S D=1,91)$ und in der Schule bei $M=7,86(S D=1,57)$. Die Angaben der Studierenden der VG unterscheiden sich nicht signifikant davon (Universität: $M=5,91(S D=1,99)$, Schule: $M=8,00(S D=1,26)$, was darauf zurückzuführen sein könnte, dass die Intervention nicht »intensiv « genug war oder dass das Thema Klassenmanagement in der VG mehr Raum einnahm als eine eingeplante videogestützte Einheit. Darüber hinaus könnten unberücksichtigte Drittvariablen potenzielle Effekte verwässern, denn das Praxissemester ist für die Studierenden häufig eine vielfältig herausfordernde und prägende Phase. Auch eine Überschätzung der Performanzen im Klassenmanagement im Prä-Test und/oder eine Unterschätzung im Post-Test ist denkbar (Stichwort: Praxisschock).

Die Daten deuten darauf hin, dass Studierende der IG im SoSe 2017 mehr hinsichtlich ihres Klassenmanagements profitierten, wenn sie nicht nur anhand von fremden, sondern auch mit eigenen Video- statt eigenen Textvignetten kriteriengeleitet klassenmanagementbezogene Handlungen reflektierten. Der Befund ist jedoch nicht statistisch signifikant. Wenngleich die Studierenden in der Untersuchung von Kramer et al. (2017) die videobasierte Reflexion als kognitiv aktivierender bewerteten als die transkriptgestützte Reflexion, so wurde dort im Vergleich eines video- gegenüber eines transkriptgestützten Seminars ebenfalls kein bedeutungsvoller Effekt für den Lernzu- 
wachs festgestellt. Vor dem Hintergrund der auch in der vorliegenden Untersuchung positiver bewerteten Lernwirksamkeit der Arbeit mit Unterrichtsvideografien gegenüber Textvignetten durch die Studierenden selbst, erscheint die Methodenwahl des Seminarkonzepts dennoch zielführend.

Die Überzeugungen der Lehramtsstudierenden zur inklusiven Bildung erscheinen relativ stabil (Vergleich vor und nach dem Praxissemester). Eine Stabilität der Einstellung zu inklusivem Lernen resumieren auch Bosse et al. (2016) in einer Längsschnittuntersuchung mit Lehrkräften über fast eineinhalb Jahre (drei Messzeitpunkte). Die in jener Untersuchung ebenfalls festgestellte Stabilität in den Selbstwirksamkeitsüberzeugungen findet sich hier nicht. Die Studierenden der IG und der VG schätzten sich am Ende des Praxissemesters wirksamer bezüglich der Umsetzung inklusiver Unterrichtspraxis ein als am Anfang (kleine bis mittlere Effekte). Unterschiede in der Berufserfahrung von Studierenden und Lehrkräften könnten dabei eine Rolle spielen. Zudem kann die Befundlage zu typischen Entwicklungsverläufen bezüglich der Selbstwirksamkeitsüberzeugungen im Studium und Praktikum insgesamt als widersprüchlich angesehen werden (Schüle, Besa, Schriek und Arnold 2017). Worauf der Zuwachs in der vorliegenden Untersuchung zurückzuführen ist, kann nicht genau bestimmt werden. Da ausgeprägte Selbstwirksamkeitsüberzeugungen einen Schutzfaktor bei der Stressbewältigung darstellen (Schmitz 2001), ist ihr Aufbau und Erhalt jedoch von elementarer Bedeutung für die LehrerInnen-Gesundheit. Eine vertiefende Analyse zu begünstigenden Faktoren (z. B. MentorInnenbetreuung) erscheint vor diesem Hintergrund bedeutsam.

Welche Rolle (Selbstwirksamkeits-) Überzeugungen bezüglich inklusiver Bildung beim Aufbau von Professionswissen - konkret im Bereich des Klassenmanagements - spielen, kann nicht abschließend beantwortet werden. Die Ergebnisse deuten darauf hin, dass Selbstwirksamkeitsüberzeugungen hinsichtlich der Umsetzung einer inklusiven Unterrichtspraxis in einem engeren Zusammenhang mit klassenmanagementbezogenen Handlungen stehen als mit Überzeugungen zu inklusiver Pädagogik. Bezüglich ihres Aufbaus könnte diesen jedoch eine Schlüsselrolle zukommen. Zugunsten einer zielführenden Weiterentwicklung von Seminarkonzepten in der LehrerInnenbildung gilt es interindividuelle Unterschiede in der Nutzung von Lerngelegenheiten zu erfassen und nutzbar zu machen.

\section{Literatur}

Avramidis, Elias, Phil Bayliss und Robert Burden. 2000. „A Survey into Mainstream Teachers' Attitudes Towards the Inclusion of Children with Special Educational Needs in the Ordinary School in one Local Education Authority.« Educational Psychology 20 (2): 191-211. https:// doi.org/10.1080/713663717

Avramidis, Elias, und Brahm Norwich. 2002. »Teachers' Attitudes towards Integration/Inclusion: A Review of the Literature." European Journal of Special Needs Education 17 (2): 129-147. https://doi.org/10.1080/08856250210129056 
Baker, Pamela H. 2005. »Managing Student Behavior: How Ready Are Teachers to Meet the Challenge?« American Secondary Education 33 (3): 51-64.

Bandura, Albert. 1977. »Self-efficacy: Toward a Unifying Theory of Behavioral Change.« Psychological Review 84 (2): 191-215. https://doi.org/10.1037/0033-295X.84.2.191

Baumert, Jürgen, und Mareike Kunter. 2006. »Stichwort: Professionelle Kompetenz von Lehrkräften.«Zeitschrift für Erziehungswissenschaft 9 (4):469-520. https://doi.org/10.1007/s1 1618-006 $-0165-2$

Blömeke, Sigrid, Jan-Eric Gustafsson und Richard J. Shavelson. 2015. „Beyond Dichotomies.» Zeitschrift für Psychologie 223 (1): 3-13. https://doi.org/10.1027/2151-2604/a000194

Blüthmann, Irmela, Diemut Ophardt, Felicitas Thiel und Gabriele Felsberger. 2011. „Wissenserwerb zum Thema Klassenmanagement im Lehramtsstudium. Individuelle und studiebezogene Einflussfaktoren. Unterrichtswissenschaft - Zeitschrift für Lernforschung 39 (4): 290-309.

Bosse, Stefanie, Thorsten Henke, Christian Jäntsch, Jennifer Lambrecht, Miriam Vock und Nadine Spörer. 2016. »Die Entwicklung der Einstellung zum inklusiven Lernen und der Selbstwirksamkeit von Grundschullehrkräften.« Empirische Sonderpädagogik 8 (1): 103-116.

Bromfield, Carolyn. 2006. »PGCE Secondary Trainee Teachers \& Effective Behaviour Management. An evaluation and commentary."Support for Learning 21 (4): 188-193. https://doi.org/10. $1111 /$ j.1467-9604.2006.00430.x

Brophy, Jere. 2006. »History of Research on Classroom Management." In Handbook of Classroom Management: Research, Practice, and Contemporary Issues, herausgegeben von Carolyn M. Evertson und Carol S. Weinstein, 17-43. Mahwah, NJ, US: Lawrence Erlbaum Associates Publishers.

Cohen, Jacob. 1988. Statistical Power Analysis for the Behavioral Sciences. Hillsdale, NJ: Erlbaum.

de Boer, Anke, Sip J. Pijl und Alexander Minnaert. 2011. »Regular Primary Schoolteachers' Attitudes towards Inclusive Education: A Review of the Literature.« International Journal of Inclusive Education 15 (3): 331-353. https://doi.org/10.1080/13603110903030089

Doyle, Walter. 2006. »Ecological Approaches to Classroom Management.» In Handbook of Classroom Management: Research, Practice, and Contemporary Issues, herausgegeben von Carolyn M. Evertson und Carol S. Weinstein, 97-125. Mahwah, NJ, US: Lawrence Erlbaum Associates Publishers.

Evertson, Carolyn M., und Carol S. Weinstein. 2006. Handbook of Classroom Management: Research, Practice, and Contemporary Issues. Mahwah, NJ, US: Lawrence Erlbaum Associates Publishers.

Feyerer, Ewald. 2014. „Einstellungen und Haltungen zur inklusiven Schule.« Österreichische pädagogische Zeitschrift Erziehung und Unterricht 164 (3-4): 219-227.

Feyerer, Ewald, Harald Reibnegger, Petra Hecht, Claudia Niedermair, Katharina Soukup-Altrichter, Christine Plaimauer, Eva Prammer-Semmler, Irene Moser und Sabine Bruch. 2016. „SACIER/TEIP - Skala für Einstellungen, Haltungen und Bedenken zu Inklusiver Pädagogik/Skala zu Lehrer/innenwirksamkeit in Inklusiver Pädagogik [Fragebogen].» In Leibniz-Zentrum für Psychologische Information und Dokumentation (ZPID), Elektronisches Testarchiv (PSYNDEX TestsNr. 9007118). Trier: ZPID. https://doi.org/10.23668/psycharchives.454

Forlin, Chris, Chris Earle, Tim Loreman und Umesh Sharma. 2011. »The Sentiments, Attitudes and Concerns about Inclusive Education Revised (SACIE-R) Scale for Measuring Pre-service Teachers' Perceptions about Inclusion. «Exceptionality Education International 21 (3): 50-65.

Fuchs, Lynn S., Douglas Fuchs und Norris Bishop. 1992. »Instructional Adaptation for Students at Risk." The Journal of Educational Research 86 (2): 70-84. https://doi.org/10.1080/00220671. 1992.9941143

Gebauer, Miriam M. 2013. "Selbstwirksamkeitsüberzeugungen von Lehrenden« In Determinanten der Selbstwirksamkeitsüberzeugung von Lehrenden: Schulischer Berufsalltag an Gymnasien 
und Hauptschulen, herausgegeben von ders., 41-66. Wiesbaden: Springer. https://doi.org/ 10.1007/978-3-658-00613-6_3

Gehrmann, Marie-Luise, Iryna Karpyuk, Tim Linka, Erin Gerlach, Hendrik Lohse-Bossenz und Satyam A. Schramm. in Vorbereitung. »Entwicklung und Überprüfung eines Selbsteinschätzungsbogens von Performanzen im Klassenmanagement von (angehenden) Lehrkräften.«

Ghaith, Ghazi, und Hussein M. Yaghi. 1997. »Relationships among Experience, Teacher Efficacy, and Attitudes toward the Implementation of Instructional Innovation." Teaching and Teacher Education 13 (4): 451-458. https://doi.org/10.1016/S0742-051X(96)00045-5

Gold, Bernadette, Stephan Förster und Manfred Holodynski. 2013. »Evaluation eines videobasierten Trainingsseminars zur Förderung der professionellen Wahrnehmung von Klassenführung im Grundschulunterricht« Zeitschrift für Pädagogische Psychologie 27 (3): 141-155. https:// doi.org/10.1024/1010-0652/a000100

Hattie, John. 2009. Visible Learning: A Synthesis of over 800 Meta-Analyses Relating to Achievement. London: Routledge.

Havers, Norbert (2015): »Kann Microteaching die Praxisrelevanz von Lehrerbildung verbessern?« In Auf die Lehrperson kommt es an? Beiträge zur Lehrerbildung nach John Hatties »Visible Learning", herausgegeben von Susanne Lin-Klitzing, David Di Fuccia und Roswitha StenglJörns, 161-174. Bad Heilbrunn: Julius Klinkhardt.

Hecht, Petra, Claudia Niedermair und Ewald Feyerer. 2016. „Einstellungen und inklusionsbezogene Selbstwirksamkeitsüberzeugungen von Lehramtsstudierenden und Lehrpersonen im Berufseinstieg. Messverfahren und Befunde aus einem Mixed-Methods-Design."Empirische Sonderpädagogik 8 (1): 86-102.

Helmke, Andreas. 2015. Unterrichtsqualität und Lehrerprofessionalität. Diagnose, Evaluation und Verbesserung des Unterrichts. Bobingen: Klett und Kallmeyer.

Hellermann, Christina, Bernadette Gold und Manfred Holodynski. 2015. »Förderung von Klassenführungsfähigkeiten im Lehramtsstudium. Die Wirkung der Analyse eigener und fremder Unterrichtsvideos auf das strategische Wissen und die professionelle Wahrnehmung."Zeitschrift für Entwicklungspsychologie und Pädagogische Psychologie 47 (2): 97-109. https://doi. org/10.1026/0049-8637/a000129

Jordan, Anne, Eileen Schwartz und Donna McGhie-Richmond. 2009. »Preparing Teachers for Inclusive Classrooms." Teaching and Teacher Education 25 (4): 535-542. http://dx.doi.org/10.1016/ j.tate.2009.02.010

Kleinknecht, Marc, und Jürgen Schneider. 2013. „What Do Teachers Think and Feel when Analyzing Videos of Themselves and Other Teachers Teaching?« Teaching and Teacher Education 33: 13-23. https://doi.org/10.1016/j.tate.2013.02.002

Klinzing, Hans G. 2002. »Wie effektiv ist Microteaching? Ein Überblick über fünfundzwanzig Jahre Forschung.« Zeitschrift für Pädagogik 48 (2): 194-214.

Kopp, Bärbel. 2009. »Inklusive Überzeugung und Selbstwirksamkeit im Umgang mit Heterogenität - Wie denken Studierende des Lehramts für Grundschulen?«Empirische Sonderpädagogik 1 (1): 5-25.

Kounin, Jacob S. 2006. Techniken der Klassenführung. Standardwerke aus Psychologie und Pädagogik. Reprints: Vol. 3. Münster: Waxmann.

Kramer, Charlotte, Johannes König, Gabriele Kaiser, Rudy Ligtvoet und Sigrid Blömeke. 2017. »Der Einsatz von Unterrichtsvideos in der universitären Ausbildung. Zur Wirksamkeit video- und transkriptgestützter Seminare zur Klassenführung auf pädagogisches Wissen und situationsspezifische Fähigkeiten angehender Lehrkräfte.« Zeitschrift für Erziehungswissenschaft 20 (S1): 137-164. https://doi.org/10.1007/s11618-017-0732-8

Kunter, Mareike, Thilo Kleickmann, Uta Klusmann und Dirk Richter. 2011. „Die Entwicklung pro- 
fessioneller Kompetenz von Lehrkräften.« In Forschung zur professionellen Kompetenz von Lehrkräften - Ergebnisse des Projekts COACTIV, herausgegeben von Mareike Kunter, Jürgen Baumert, Werner Blum, Uta Klusmann, Stefan Krauss und Michael Neubrand, 55-68. Münster: Waxmann.

Linka, Tim, und Marie-Luise Gehrmann. 2018. „Klassenmanagement im Schulzimmer und der Sporthalle - Entwicklung und Evaluation eines videogestützten Seminarkonzeptes in den Praxisphasen der universitären Lehrerbildung. In Potsdamer Beiträge zur Lehrerbildung und Bildungsforschung. PSI-Potsdam: Ergebnisbericht zu den Aktivitäten im Rahmen der Qualitätsoffensive Lehrerbildung (2015-2018), herausgegeben von Andreas Borowski, Antje Ehlert und Helmut Prechtl, 201-215. Universitätsverlag Potsdam.

Lohse-Bossenz, Hendrik, und Karsten Krauskopf. 2017. „Strukturiert und vernetzt - eine experimentelle Studie zur Reflexion von Unterrichtsvideos. "Vortrag gehalten auf der 82. Tagung der Arbeitsgruppe empirisch pädagogische Forschung der Deutschen Gesellschaft für Erziehungswissenschaft (DGfE), Tübingen.

Marzano, Robert J., Jana S. Marzano und Debra Pickering. 2003. Classroom Management That Works. Research-Based Strategies for Every Teacher. Alexandria, Va: Association for Supervision and Curriculum Development.

Milner, H. Richard. 2002. »A Case Study of an Experienced English Teacher's Self-efficacy and Persistence through >Crisis` Situations: Theoretical and Practical Considerations. "The High School Journal 86 (1): 28-35. https://doi.org/10.1353/hsj.2002.0020

Oliver, Regina M., Joseph H. Wehby und Daniel J. Reschly. 2011. »Teacher Classroom Management Practices: Effects on Disruptive or Aggressive Student Behavior.«Campbell Systematic Reviews 4: $1-55$.

Ophardt, Diemut, Valentina Piwowar und Felicitas Thiel. 2017. »Kompetenzen des Klassenmanagements (KODEK). Entwicklung und Evaluation eines Fortbildungsprogramms für Lehrpersonen zum Klassenmanagement« In Entwicklung von Professionalität pädagogischen Personals, herausgegeben von Cornelia Gräsel und Kati Trempler, 133-152. Wiesbaden: Springer Fachmedien Wiesbaden. https://doi.org/10.1007/978-3-658-07274-2_8

Pawelzik, Janina 2017. »Zusammenhänge zwischen Überzeugungen von Studierenden zum naturwissenschaftlichen Sachunterricht und praxisbezogenen Lerngelegenheiten: Eine Studie im Rahmen des Projektes »Integration von Theorie und Praxis - Partnerschulen (ITPP)« Inauguraldissertation, Westfälische Wilhelms-Universität Münster.

Pigge, Fred L., und Marso, Ronald N. 1997. »A Seven-year Longitudinal Multi-Factor Assessment of Teaching Concerns Development through Preparation and Early Years of Teaching.« Teaching and Teacher Education 13 (2): 225-235. https://doi.org/10.1016/S0742-051X(96)00014-5

Reusser, Kurt, und Christine Pauli. 2014. »Berufsbezogene Überzeugungen von Lehrerinnen und Lehrern." In Handbuch der Forschung zum Lehrerberuf, herausgegeben von Ewald Terhart, Hedda Bennewitz und Martin Rothland, 642-661. Münster: Waxmann.

Sadler, Jane. 2005. »Knowledge, Attitudes and Beliefs of the Mainstream Teachers of Children with a Preschool Diagnosis of Speech/Language Impairment." Child Language Teaching and Therapy 21 (2): 147-163. https://doi.org/10.1191/0265659005ct286oa

Schmitz, Gerdamarie S. 2001. „Kann Selbstwirksamkeitserwartung vor Burnout schützen? Eine Längsschnittstudie in zehn Bundesländern. Psychologie in Erziehung und Unterricht 48 (1): 49-67.

Schüle, Christoph, Kris-Stephen Besa, Josina Schriek und Karl-Heinz Arnold. 2017. »Die Veränderung der Lehrerselbstwirksamkeitsüberzeugung in Schulpraktika.« Zeitschrift für Bildungsforschung 7 (1): 23-40. https://doi.org/10.1007/s35834-016-0177-9

Schwarzer, Ralf, und Lisa M. Warner. 2014. »Forschung zur Selbstwirksamkeit bei Lehrerinnen und 
Lehrern." In Handbuch der Forschung zum Lehrerberuf, herausgegeben von Ewald Terhart, Hedda Bennewitz und Martin Rothland, 496-510. Münster: Waxmann.

Seidel, Tina, und Felicitas Thiel. 2017. "Standrads und Trends der videobasierten Lehr-Lernforschung.« Zeitschrift für Erziehungswissenschaft 20 (S1): 1-21. https://doi.org/10.1007/s11618 -017-0726-6

Sekretariat der Ständigen Konferenz der Kultusminister der Länder in der Bundesrepublik Deutschland. 2018. Sonderpädagogische Förderung in Schulen 2007 bis 2016: Dokumentation Nr. 214, Juni 2018. Zugriff 02. April 2019. https://www.kmk.org/dokumentation-statistik/statistik/ schulstatistik/sonderpaedagogische-foerderung-an-schulen.html

Sermier Dessemontet, Rachel, Valérie Benoit und Gérad Bless. 2011. "Schulische Integration von Kindern mit einer geistigen Behinderung - Untersuchung der Entwicklung der Schulleistungen und der adaptiven Fähigkeiten, der Wirkung auf die Lernentwicklung der Mitschüler sowie der Lehrereinstellungen zur Integration.«Empirische Sonderpädagogik 3 (4): 291-307.

Sharma, Umesh, Tim Loreman und Chris Forlin. 2012. »Measuring Teacher Efficacy to Implement Inclusive Practices. « Journal of Research in Special Educational Needs 12 (1): 12-21. https://doi. org/10.1111/j.1471-3802.2011.01200.x

Sharma, Umesh, und Laura Sokal. 2016. "Can Teachers' Self-Reported Efficacy, Concerns, and Attitudes Toward Inclusion Scores Predict Their Actual Inclusive Classroom Practices?« Australasian Journal of Special Education 40 (01): 21-38. https://doi.org/10.1017/jse.2015.14

Wallace, Teri, Amy Reschly Anderson, Tom Bartholomay und Susan Hupp. 2002. »An Ecobehavioral Examination of High School Classrooms That Include Students with Disabilities." Exceptional Children 68 (3), 345-359. https://doi.org/10.1177/001440290206800304

Wang, Margaret C., Geneva D. Haertel und Herbert J. Walberg. 1994. »What Helps Students Learn? Spotlight on Student Success."Educational Leadership 51: 74-79.

Woodcock, Stuart, und Andrea Reupert. 2012. »A Cross-sectional Study of Student Teachers' Behaviour Management Strategies throughout their Training Years." Australian Educational Researcher 39 (2): 159-172. http://dx.doi.org/10.1007/s13384-012-0056-x

Woolfolk, Anita E., Barbara Rosoff und Wayne K. Hoy. 1990. »Teachers' Sense of Efficacy and their Beliefs about Managing Students." Teaching and Teacher Education 6 (2): 137-148. https:// doi.org/10.1016/0742-051X(90)90031-Y

\section{Die Autorlnnen}

Marie-Luise Gehrmann, M. A., ist wissenschaftliche Mitarbeiterin an der Universität Potsdam am Lehrstuhl Inklusionspädagogik - Förderschwerpunkt emotionale und soziale Entwicklung. Ihre Arbeitsschwerpunkte sind: Gefühls- und Verhaltensstörungen des Kindes- und Jugendalters (Diagnostik, Prävention, Intervention), Peereinflussprozesse im Bereich emotionaler und sozialer Entwicklung sowie Förderung des Klassenmanagements in der Lehrerlnnenbildung mittels Micro-teaching.

Kontakt:mgehrman@uni-potsdam.de

Satyam Antonio Schramm, Prof. Dr. Dipl.-Psych., ist Professor für Inklusionspädagogik - Förderschwerpunkt emotionale und soziale Entwicklung an der Universität Potsdam. Seine Arbeitsschwerpunkte sind: Gefühls- und Verhaltensstörungen des Kindes- und Jugendalters, Entwicklungspsychopathologie des Kindes- und Jugendalters, Frühkindliche Sprachentwicklung sowie die Evaluation von Präventions- und Interventionskonzepten.

Kontakt: satyam.schramm@uni-potsdam.de 